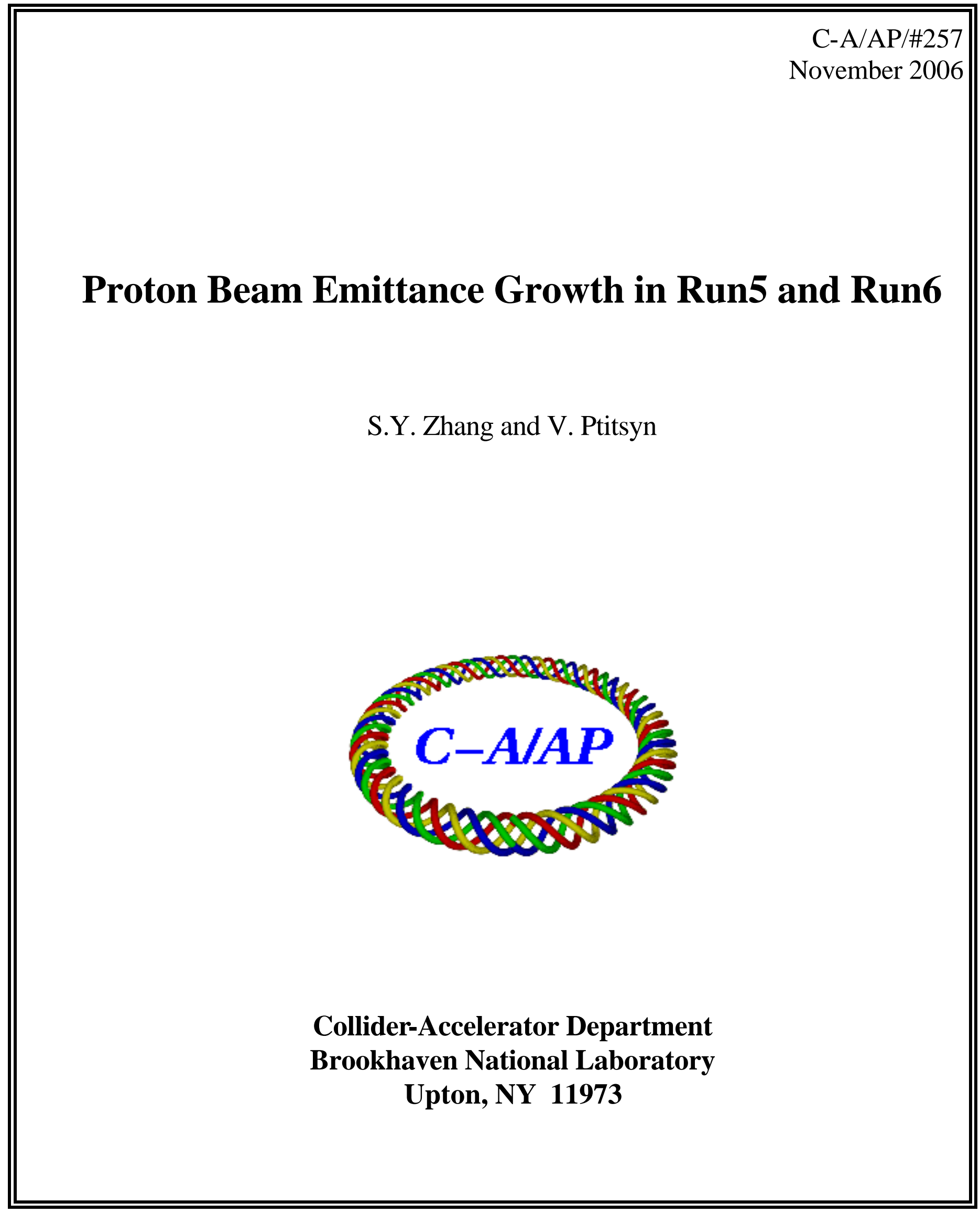




\title{
Proton Beam Emittance Growth in Run5 and Run6
}

\author{
S.Y. Zhang and V. Ptitsyn
}

\begin{abstract}
The proton beam emittance growth in RHIC Run5 and Run6 has a dependence on the dynamic pressure rise, which is caused by the electron cloud and peaked at the end of the beam injection and early acceleration. At store, the electron cloud is not significant, it seems that the emittance growth is dominated by the beam-beam effect.
\end{abstract}

\section{Introduction}

The application of $200 \mathrm{~m}$ non-evaporable getter (NEG) coated beam pipe in 2004 helped in reducing the highest dynamic pressure rise in the Q3-Q4 sections, namely Bo2 and Bi8 in Blue ring, and Yo1 in Yellow ring [1]. This allowed more bunches with higher intensity in Run5 operation, i.e., up to 110 bunches with $0.9 \times 10^{11}$ protons per bunch, compared to 55 bunches with $0.7 \times 10^{11}$ protons per bunch in Run4.

With much higher beam intensities, the emittance growth was observed in Run5. The intensity threshold of the emittance growth was approximately $140 \times 10^{11}$ protons in total of two beams. As a result, the highest intensity beam did not yield highest luminosity. For example, Fill 7327 with 104 bunches and $0.90 \times 10^{11}$ protons per bunch gave rise to average luminosity of PHENIX and STAR as $10.2 \times 10^{30} / \mathrm{cm}^{2} s$, but Fill 7264 with 82 bunches and $0.87 \times 10^{11}$ protons per bunch had higher luminosity of $11.1 \times 10^{30} / \mathrm{cm}^{2} \mathrm{~s}$.

With additional $180 \mathrm{~m}$ NEG pipes installed and also probably due to changes of machine configurations, the intensity threshold of beam emittance growth in Run6 has increased to at least $260 \times 10^{11}$ protons total. Average luminosity in Run6 was increased by factor 3 from Run5. The typical bunch intensity in Run5 was $0.9 \times 10^{11}$ protons and emittance was $25 \pi \mu m$, at early 
store. In Run6, typical bunch intensity of $1.3 \times 10^{11}$ protons and emittance of $17 \pi \mu m$ show that the luminosity improvement of factor 2 came from the intensity increase, and another factor of 1.5 came from the emittance control.

In Run7, only $45 \mathrm{~m}$ NEG pipes will be installed, the impact will be less than Run5 and Run6. The effect of machine configuration on pressure rise and emittance growth needs to be investigated [2]. Meanwhile, a better understanding of the emittance growth would be beneficial to the further improvement of machine luminosity.

This article discusses several aspects of the RHIC proton beam emittance growth observed in Run5 and Run6, as well as its mechanism.

\section{Proton beam emittance growth}

In Fig.1, the proton beam emittances of the last 44 fills in 2005 and the last 90 fills in $2006100 \mathrm{GeV}$ polarized proton run are shown against the total beam intensity. The emittance is calculated using the zero degree calorimeter (ZDC) coincident rates, taking average of the experiments PHENIX and STAR. The data are taken at the softev-physics event in Run5, and at 1.5 hours after the accramp event in Run6. These are similar in the timing during the production of a physics store, representing the peak luminosity.

The emittance growth in Run5 is larger than that in Run6, therefore, some features of the emittance growth can be better identified. On the other hand, in Run6, the ionization profile monitor (IPM) is improved, and some beam study is carried out. At below, the beam emittance growth is discussed using Run5, and/or Run6 data.

\subsection{Dependence on bunch number, bunch intensity, and total beam intensity}

In Fig.2, the emittance growth dependence on bunch intensity and bunch number is shown for the Run5 data.

No emittance growth dependence on bunch intensities from $0.61 \times 10^{11}$ to $0.91 \times 10^{11}$ protons can be identified in Fig.2a. This suggests that the beam-beam effect, both head-on and long range, was not a dominant factor in beam emittance growth. The absence of significant emittance growth in Run6, which has comparable peak beam-beam parameter with Run5, seems agreeable with this conclusion. 


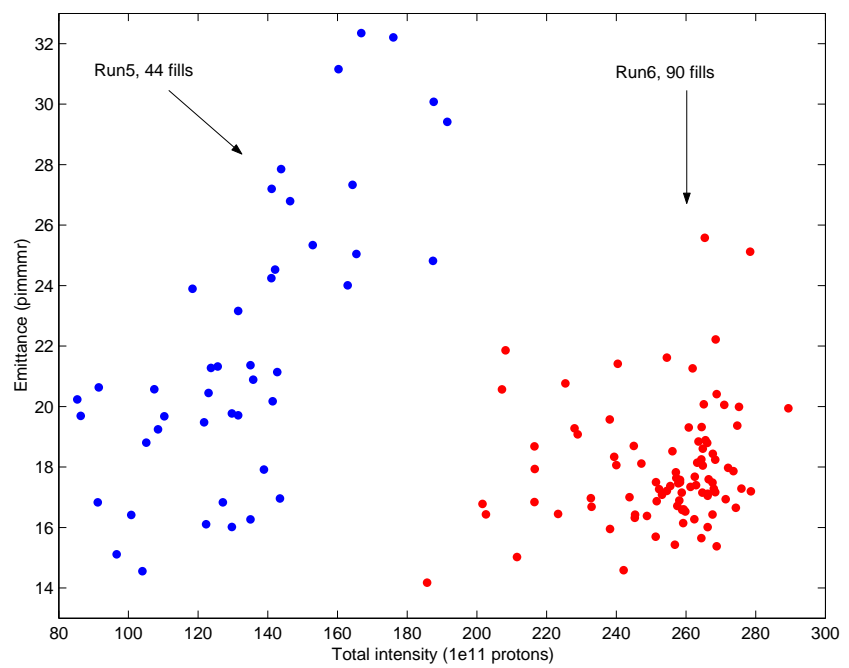

Figure 1: Normalized beam emittance versus total beam intensity in 2005 and $2006100 \mathrm{GeV}$ polarized proton runs. The emittance is calculated from the average ZDC coincident rates of the experiments PHENIX and STAR, at early store.
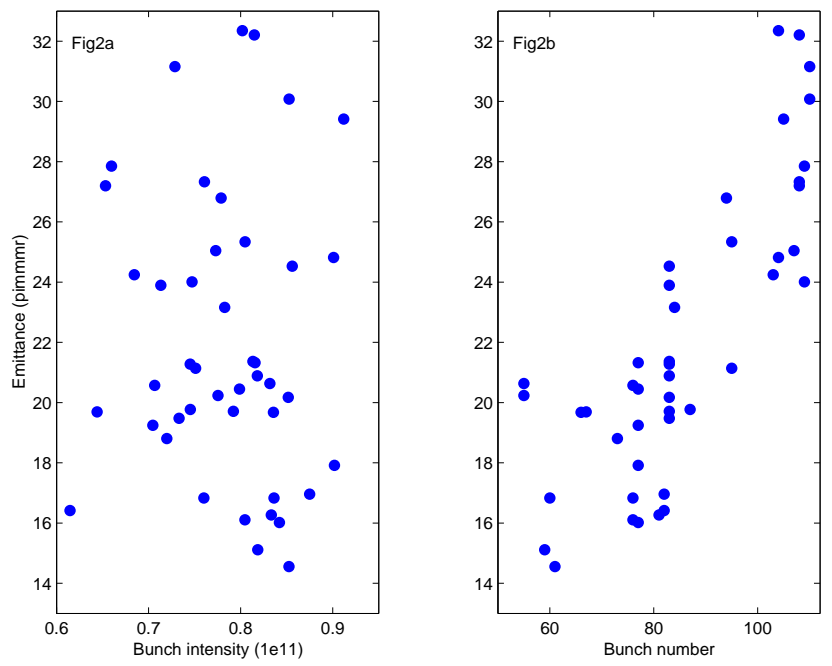

Figure 2: Emittance growth dependence on bunch intensity and bunch number for Run5. The dependence of the emittance growth on the bunch number can be clearly identified. 
In Fig.2b, a clear dependence of the emittance growth on the bunch number is shown. The bunch number is from 55 bunches (average bunch spacing $216 \mathrm{~ns}$ ) to 110 bunches (bunch spacing $108 \mathrm{~ns}$ ). The Run5 emittance growth data are replotted in Fig.3a verses the total beam intensity, which is the product of bunch intensity and bunch number. In a period of two weeks, the AGS radial steering problem caused larger beam emittance than usual. This problem was corrected shortly before the end of the run. The fills during this period are marked by black dots. In Fig.3b the beam emittance with the AGS problem is shifted down by $4 \pi \mu m$, and the intensity threshold of the emittance growth can be better seen.

The emittance growth dependence on the beam intensity at RHIC is similar to that observed at KEKB [3], and PEPII [4]. In both machines, there is an intensity threshold of the emittance growth. Beyond this threshold, the beam size, which is proportional to a square root of the emittance, grows linearly with the intensity. The RHIC emittance growth in Run5 shows a threshold at about $140 \times 10^{11}$ protons total. Due to limited data, the linear beam size growth above the threshold can only be approximately identified, not as clearly as the electron machines.

\subsection{Dependence on dynamic pressure rise}

The dynamic pressure rise in RHIC proton cycles is usually peaked at the end of the beam injection and then at the $\mathrm{RF}$ voltage ramping. Immediately after RF voltage ramping, the energy acceleration is started. The pressure rise is then gradually reduced, and becomes insignificant at the store.

The beam emittance growth has a dependence on the dynamic pressure rise observed in the warm sections for Run5 and Run6. All fills with small emittance growth have low pressure rise, and all fills with high pressure rise have large emittance growth. Using the average pressure rise measured at the middle of all 24 Q3-Q4 straight sections, typical fills are illustrated in Table 1. Fill 7250 in Run5 and Fill 7909 in Run6 have little emittance growth, and Fill 7325 in Run5 has large emittance growth. The pressure rise of 7325 is about a factor of 10 higher than 7250 and 7909 . 

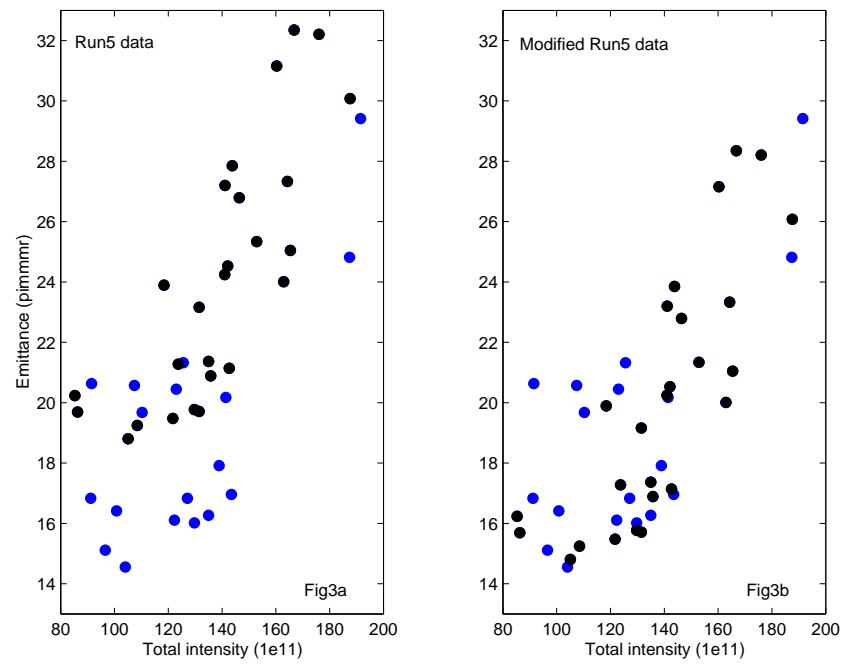

Figure 3: The Run5 emittance versus the total beam intensity is shown in Fig.3a. The fills with the AGS problem are in black dots. In Fig.3b, these are shifted down by $4 \pi \mu m$, to identify the intensity threshold.
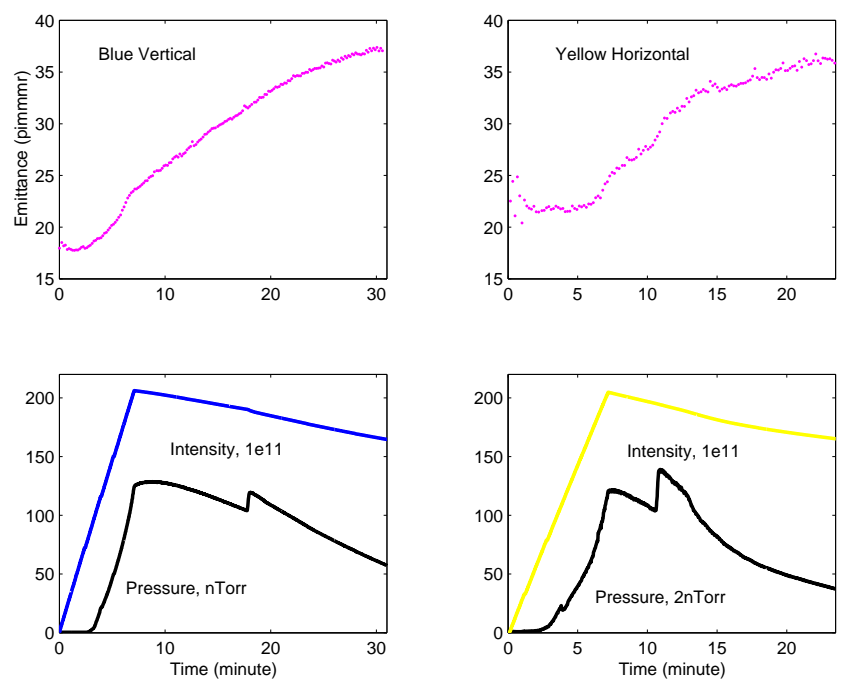

Figure 4: Beam emittance growth is compared with dynamic pressure rise. Blue vertical and Yellow horizontal IPM emittances are shown with the pressure rise in Blue and Yellow rings, respectively. The first peak pressure rise is at the end of injection, and the second peak is at the RF voltage ramping. 


$\begin{array}{cccc}\text { Fill } & 7250 \text { (Run5) } & 7325 \text { (Run5) } & 7909 \text { (Run6) } \\ \text { Total intensity, } 10^{11} & 130 & 190 & 269 \\ \text { Emittance, } \pi \mu m & 16.0 & 29.4 & 15.4 \\ \text { ZDC coincident rate, } \mathrm{kHz} & 3.98 & 3.46 & 12.65 \\ \text { e. pressure rise, } 10^{-8} \text { Torr } & 0.35 & 3.86 & 0.37\end{array}$

Table 1: Typical fills of emittance growth in Run5 and Run6, the average ZDC of PHENIX and STAR, and the average pressure rise.

In a beam study during Run6 (Fill 7935), 110 bunches with $1.9 \times 10^{11}$ protons per bunch were injected, then the RF voltage was increased from $160 \mathrm{kV}$ to $300 \mathrm{kV}$ to shorten the bunch. The beam emittance growth observed by the IPM shows correlations with the pressure rise. In Fig.4, the Blue vertical and Yellow horizontal IPM emittance data are compared with the pressure rises, which are the average of 4 Q3-Q4 straight sections (with higher pressure rise than others) in Blue and Yellow rings, respectively. The emittance measurement using the polarimeter target is in agreement with this IPM measurement.

The average pressure rise in all 24 Q3-Q4 sections in this beam study is $2.66 \times 10^{-8}$ Torr, comparable with the Fill 7325 in Run5, which had large emittance growth.

With this pressure rise, the beam emittance growth from the IPM data is about $15 \pi \mu m$ in 20 minutes at the beam injection energy.

In the following, several other observations are shown to demonstrate the emittance growth dependence on the pressure rise.

\subsubsection{Timing of the emittance growth}

If the pressure rise is indeed responsible to the beam emittance growth, then the emittance growth should take place mainly at the injection and early acceleration.

In Fig.5, the average Blue and Yellow IPM horizontal data taken at 1 minute after the accramp event (the start of energy acceleration) is compared with the beam emittance calculated using ZDC coincident rate for Run5. The correlation shows that when the IPM emittance increases from $10 \pi \mu m$ to 28 $\pi \mu m$, the emittance from ZDC is increased from $14 \pi \mu m$ to $32 \pi \mu m$. Since the IPM emittance is measured at 1 minute after accramp and ZDC emittance at 


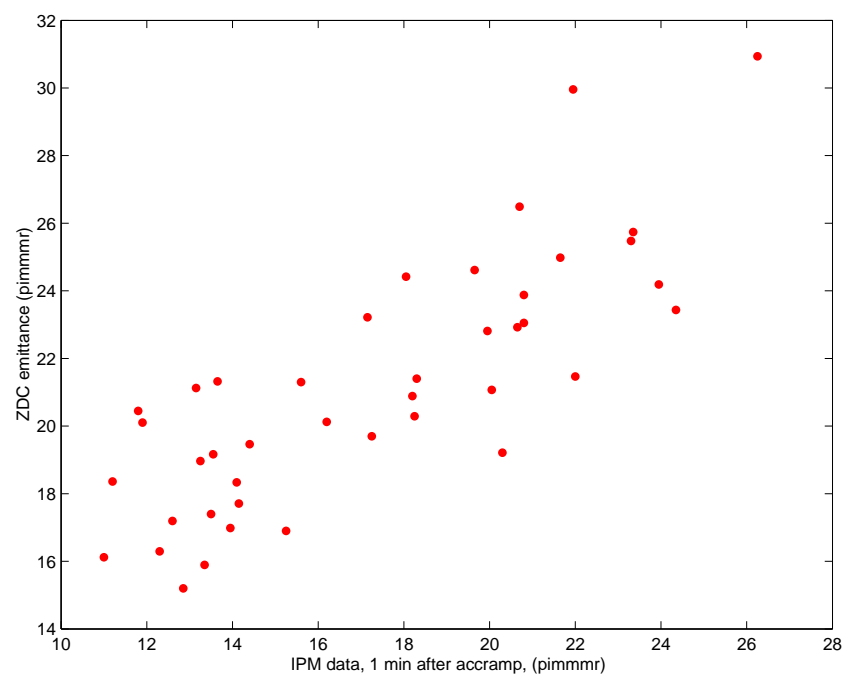

Figure 5: Correlation of the average Blue and Yellow IPM horizontal emittance measurement at the middle of the energy ramp with the emittance calculated from the ZDC coincident rate for Run5 data.
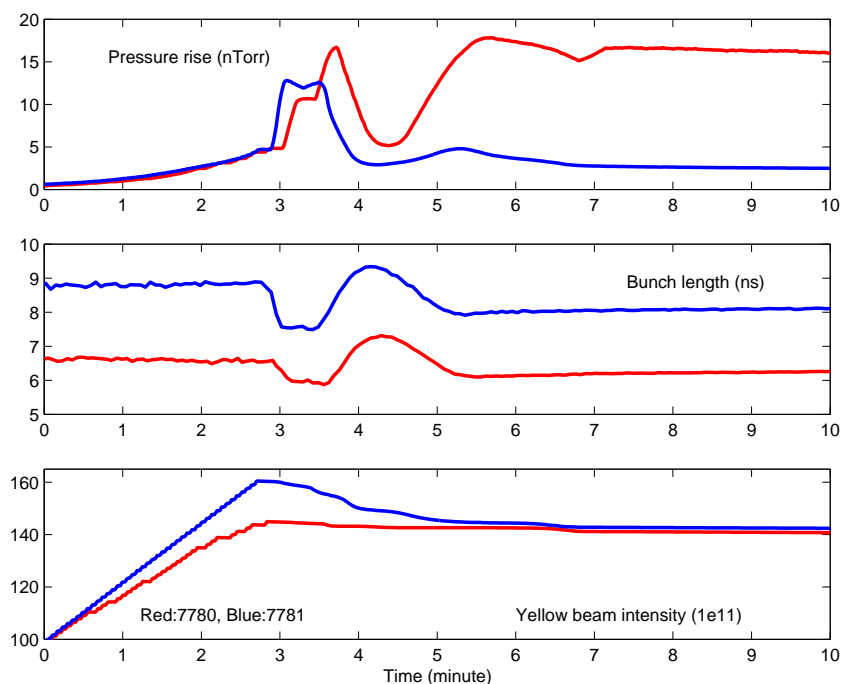

Figure 6: Beam intensity, bunch length, and pressure rise of fills 7780 (red) and 7781 (blue). The longer bunch of 7781 induces lower pressure rise, which can be seen from the later part with the same intensity. The trade-off of the intensity and bunch length in terms of pressure rise can be seen from the early part. 
the early store, this correlation suggests that a significant emittance growth has occurred before the middle of the energy ramp (entire energy ramp is about 2.2 minutes), including the beam injection. This is consistent with the time period of highest dynamic pressure rise in the machine cycle, i.e. at the end of the injection and early acceleration.

IPM emittance measurement is affected by several factors, including the calibration with the $\beta$ function at the location of the IPM, which is less reliable, especially during the energy ramp. These factors, however, should not affect the fact identified in Fig.5, i.e., a significant emittance growth has happened before the middle of the energy ramp for the high beam intensity.

\subsubsection{Bunch length effect}

One of the most significant effect of the bunch length is the dynamic pressure rise. In Fig.6, it is shown that for same intensity, shorter bunches induce higher pressure rise.

In Run6, the machine luminosity was closely watched and compared with the then 'golden', or best, fill. The cluster in the intensity-emittance plane shown in Fig.1, consisted with fills during last 7 weeks, suggests that the machine limit has been reached and only less significant luminosity improvement is possible. The bunch length dependence of the emittance growth then becomes noticeable in operation. During this time period, the FWHM bunch length varies between $6.5 \mathrm{~ns}$ to $8.5 \mathrm{~ns}$ (prior to the $\mathrm{RF}$ voltage ramping). It was found that almost all 'golden' fills have longer bunches, around 8 ns. The lower dynamic pressure rise associated with longer bunches may have caused smaller emittance growth in these fills, and hence a higher machine luminosity.

Another indication that shorter bunches cause problems for transverse emittance came during the attempts to use the quad pumping to reduce the bunch length in RHIC. The fills with quad pumping had lower luminosity for same beam intensities. In Fig.7, the PHENIX ZDC, the pressure rise, and the beam intensities are shown for Fill 7856, without quad pumping, and 7860 , with quad pumping. The effective bunch length in 7860 was reduced by more than $20 \%$, which caused much higher pressure rise. The luminosity of 7860 is clearly lower than 7856 . 

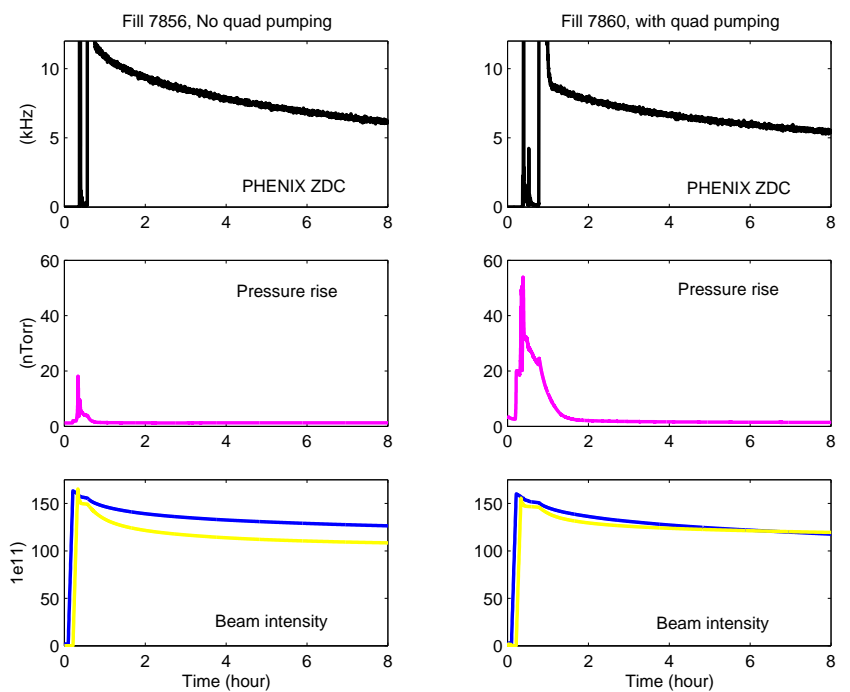

Figure 7: Fill 7856 without quad pumping, and 7860 with quad pumping. The PHENIX ZDC, the sum of pressure rise in 4 each Blue and Yellow Q3-Q4 sections with less NEG pipes, and the beam intensities are shown.

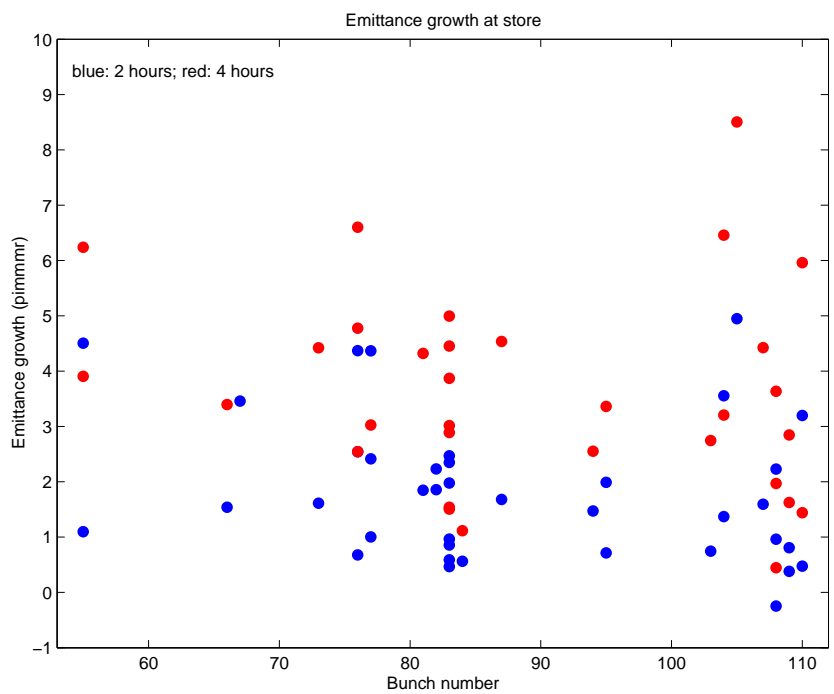

Figure 8: Beam emittance growth in store versus bunch number for Run5. The blue and red dots are for 2 hours and 4 hours of the emittance growth, respectively. No dependence can be identified. 


\subsection{Emittance growth at store}

If the beam emittance growth depends mainly on the dynamic pressure rise, then a less emittance growth is expected at store, due to insignificant pressure rise there. The emittance growth at store is indeed slower. Furthermore, the emittance growth dependence on the bunch number, which is shown in Fig.2b, is not observed at store. In Fig.8, the emittance growth in 2 hours and 4 hours during the store for Run 5 is shown against the bunch number. No dependence can be identified.

The emittance growth during the store in Run5 and Run6, however, shows some dependence on the beam-beam parameter, which is shown in Fig.9. The beam-beam parameter is scaled by $M N_{b} / \epsilon_{N}$, where $M$ is the number of collisions, $N_{b}$ is the bunch intensity, and $\epsilon_{N}$ is the normalized emittance. Bunch intensity in Run6 is increased to $1.3 \times 10^{11}$ protons from $0.9 \times 10^{11}$ protons in Run5, however, the number of collisions in Run6 is 2 and it is 3 in Run5. The peak beam-beam parameter in Run5 and Run6 is, therefore, similar. The overall emittance growth in store has similar dependence on beam-beam parameter in Run5 and Run6, regardless the different collisions, bunch intensities, and emittances.

Note that the bunch lengthening during the store provides false contribution into the transverse emittance calculated from the coincident rates. It happens because of the hour-glass effect, since the proton bunch length is of the order of the $\beta^{*}$. However, this factor should not contribute into the dependence of the emittance growth on the beam-beam parameter, shown in Fig.9 (unless the bunch lengthening itself is caused by the beam-beam interactions).

The beam intensity lifetime is not significantly affected by the beam-beam parameter, and the luminosity lifetime is mainly depending on the emittance growth during the store. In Fig.10, the average luminosity lifetime of early 4 hours in store is shown against the beam-beam parameter. Given the same 2 collisions, if the emittance keeps the same, then $50 \%$ increase of the beam intensity in Run7 will push the beam-beam parameter to about 0.018. If the decline of the luminosity lifetime with beam-beam effect observed in Run5 and Run6 is not improved, then it will be a problem in Run7. 


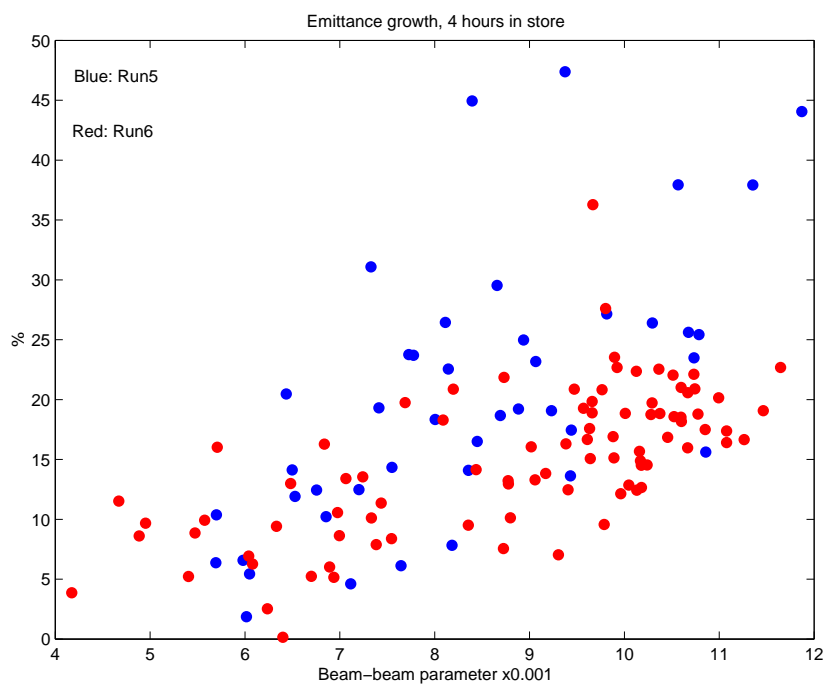

Figure 9: Beam emittance growth of 4 hours in store versus the beam-beam parameter for Run5 (blue) and Run6 (red). The bunch intensities in Run6 is about $50 \%$ higher than that in Run5, however, the number of collisions are 3 in Run5 and 2 in Run6.

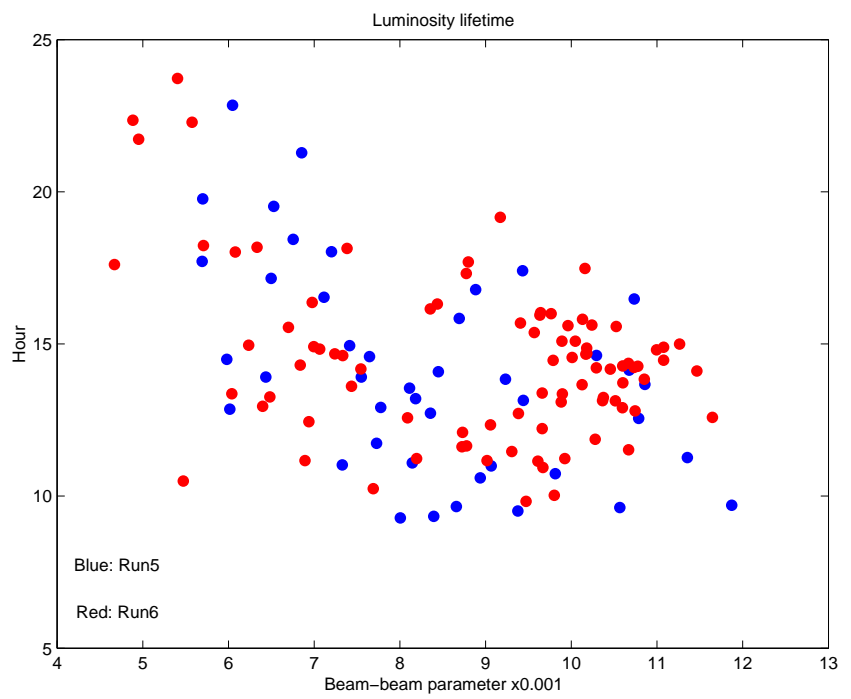

Figure 10: Average luminosity lifetime of early 4 hours in store, in Run5 and Run6. 


\section{Discussion}

For RHIC Run5 and Run6, the proton beam emittance growth at the injection and early acceleration has a dependence on dynamic pressure rise, and the slow emittance growth at store seems due to the beam-beam effect. The emittance growth during the acceleration and early store is affected by both the pressure rise and beam-beam effect, with other factors remain to identify.

The emittance growth associated with the dynamic pressure rise is affecting the peak machine luminosity, and it is very important to understand the mechanism of this growth. The direct effect of the pressure rise on the beam, i.e., the beam-gas effect, cannot explain the emittance growth. On the other hand, since the dynamic pressure rise at RHIC proton operations is caused by the electron cloud, the electron-beam interaction during the bunch passage is likely causing the emittance growth.

Electron cloud induced beam emittance growth has been machine luminosity limits at KEKB and PEPII [3,4], where the emittance growth dependence on the beam intensity is similar to that observed at the RHIC. These include the existence of the intensity threshold of the emittance growth, and the linear beam size growth above the threshold at high intensities. In both B-factories and the RHIC, the electron multipacting has been detected and the tune shift associated with the electron cloud are observed.

An important feature of the emittance growth in all these machines is that it is not accompanied with the beam instability. The emittance growth below the instability threshold is currently under intense study $[5,6]$. Simulations suggest the electron pinch caused nonlinear incoherent tune shift along the bunch and the excited resonances might be causes of the emittance growth.

Compared with the emittance growth caused by the beam instability (often observed in RHIC operations), the one below the instability threshold is much slower [6]. This slow emittance growth has already taken significant effect in machine luminosities at RHIC, and the impact at the LHC at CERN is expected to be larger. The LHC will have electron multipacting with 25 $n s$ bunch spacing at the long store, even after beam scrubbing. As comparison, the RHIC electron multipacting is insignificant at the store, and the B-factories have short stores, around 1 hour. 


\section{References}

[1] S.Y. Zhang, H.C. Hseuh, W. Fischer, H. Huang, T. Roser, NEG Coating Application at RHIC, C-A/AP/220, Oct. 2005.

[2] S.Y. Zhang, H.C. Hseuh, C. Montag, V. Ptitsyn, D. Trbojevic, Pressure Rise in Run6 and Related Issues, C-A/AP/233, Apr. 2006.

[3] H. Fukuma, ECLOUD’04, Napa, CA, 2004.

[4] F-J. Decker, ECLOUD'02, Geneva, Switzerland, 2002.

[5] M. Furman, HB-2006, Tsukuba, Japan, 2006.

[6] E. Benedetto, HB-2006, Tsukuba, Japan, 2006. 